Provided for non-commercial research and education use. Not for reproduction, distribution or commercial use.

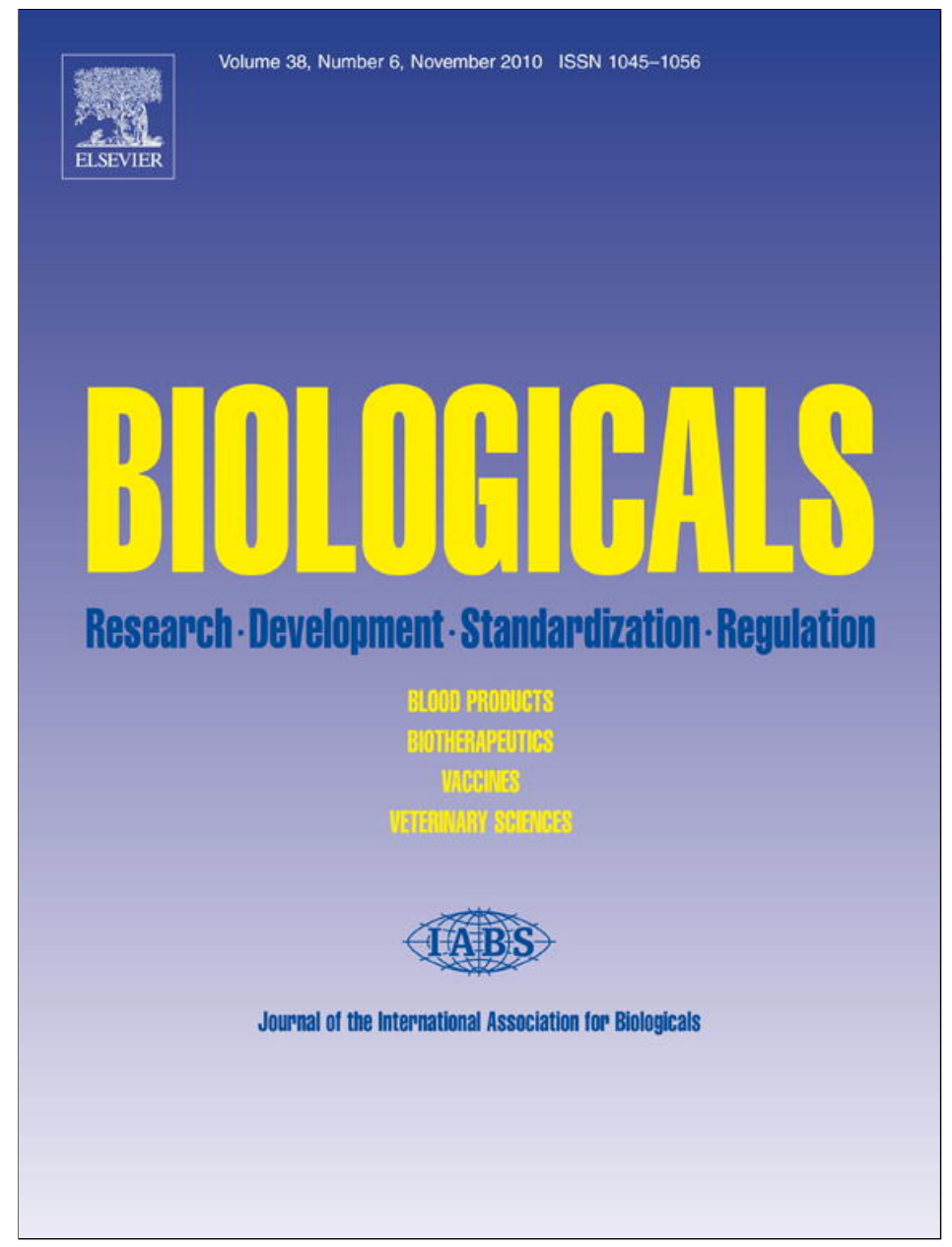

This article appeared in a journal published by Elsevier. The attached copy is furnished to the author for internal non-commercial research and education use, including for instruction at the authors institution and sharing with colleagues.

Other uses, including reproduction and distribution, or selling or licensing copies, or posting to personal, institutional or third party websites are prohibited.

In most cases authors are permitted to post their version of the article (e.g. in Word or Tex form) to their personal website or institutional repository. Authors requiring further information regarding Elsevier's archiving and manuscript policies are encouraged to visit:

http://www.elsevier.com/copyright 


\title{
Intravenous administration of equine-derived whole IgG antivenom does not induce early adverse reactions in non-envenomed horses and cows
}

\author{
Ricardo Estrada $^{\mathrm{a}}$, María Herrera ${ }^{\mathrm{a}}$, Álvaro Segura ${ }^{\mathrm{a}}$, Javier Araya ${ }^{\mathrm{b}}$, Carlos Boschini $^{\mathrm{b}}$, José María Gutiérrez $^{\mathrm{a}}$, \\ Guillermo León ${ }^{a}$,* \\ a Instituto Clodomiro Picado, Facultad de Microbiología, Universidad de Costa Rica, San José, Costa Rica \\ ${ }^{\mathrm{b}}$ Estación Experimental de Ganado Lechero Alfredo Volio Mata, Facultad de Ciencias Agroalimentarias, Universidad de Costa Rica, San José, Costa Rica
}

\section{A R T I C L E I N F O}

\section{Article history:}

Received 5 January 2010

Received in revised form

20 July 2010

Accepted 11 August 2010

\section{Keywords:}

Snakebite

Antivenom

Early adverse reactions

Caprylic acid

Cow

Horse

\section{Introduction}

Snake antivenoms are immunotherapeutic products used to treat snakebite envenoming. The active substances of these formulations are immunoglobulins, or immunoglobulin fragments, purified from the plasma of animals immunized with snake venoms [1,2]. Although therapeutic effectiveness of antivenoms has been widely demonstrated, the occurrence of early adverse reactions associated with their administration remains a matter of concern and an aspect that must be improved in envenoming immunotherapy [3].

Early adverse reactions, such as nausea, headache, dizziness, fever, vomiting, diarrhea, urticaria, generalized rash, tachypnea, tachycardia, bronchospasm or hypotension, may occur during the first $2 \mathrm{~h}$ after administration of antivenom. Depending of their underlying pathogenic mechanism, early adverse reactions can be truly IgE-mediated anaphylactic reactions or, more frequently, anaphylactoid reactions [1].

\footnotetext{
* Corresponding author. Tel.: +506 22293135; fax: +506 22920485.

E-mail address: guillermo.leon@ucr.ac.cr (G. León).
}

Anaphylactic reactions are rarely reported during administration of antivenoms [4]. These reactions occur in patients previously exposed to heterologous immunoglobulins and correspond to IgEmediated type I hypersensitivity. During the first exposure, sensitization occurs and IgE antibodies against antivenom proteins are produced. Then, during a subsequent exposure, the cross-linking of these IgE antibodies promotes the degranulation of mast cells, with the release of vasoactive substances that mediate the anaphylactic reaction [5].

In contrast to anaphylatic reactions, anaphylactoid reactions are observed in patients who have not been previously exposed to heterologous protein [1]. The incidence of anaphylactoid reactions in human patients receiving horse-derived antivenoms ranges between $5 \%$ and $80 \%$, depending on the physicochemical quality of the product administered [3].

The mechanisms underlying anaphylactoid reactions are not completely clear at present. They have been related to activation of the complement system by two different mechanisms: 1) the action of immunoglobulin aggregates formed during the process of plasma fractionation for antivenom production [6], and 2) the formation of immune complexes between immunoglobulins that constitute the antivenom and natural anti-equine heterophilic antibodies present in the plasma of the patients $[7,8]$. However, there is not a strict 
correlation between the in vitro anticomplementary activity of antivenoms and the incidence of anaphylactoid reactions at the clinical setting [9-13]. Thus, the mechanisms of analphylactoid reactions remain unclear.

In Costa Rica, the Instituto Clodomiro Picado produces polyspecific antivenom for the treatment of envenomings induced by snakes of the family Viperidae in Central America. It consists of whole IgG purified from equine plasma by caprylic acid precipitation of non-IgG plasma proteins [14]. In clinical studies performed in humans, it has been determined that this formulation induces severe anaphylactic reactions in less than $0.5 \%$ of patients and anaphylactoid reactions in about $15-20 \%$ of patients when the antivenom is administered by the intravenous route [11,15]. However, there is no information documenting the incidence of adverse reactions in animals.

The present report describes a study designed to determine the incidence of early and late adverse reactions induced by equinederived whole IgG antivenom when it is intravenously administered in non-envenomed horses and cows.

\section{Materials and methods}

\subsection{Antivenom}

Polyspecific antivenom was produced by Instituto Clodomiro Picado (batch 4240108POLQ). This formulation is constituted by whole IgG molecules. Immunoglobulins were purified by caprylic acid precipitation [14] from the plasma of horses immunized with venoms of the snakes Bothrops asper, Crotalus simus and Lachesis stenophrys [16]. The antivenom was formulated at a total protein concentration of $5.7 \mathrm{~g} / \mathrm{dL}$, with $0.85 \mathrm{~g} / \mathrm{dL} \mathrm{NaCl}, 0.25 \mathrm{~g} / \mathrm{dL}$ phenol, and $\mathrm{pH} 7.2$.

\subsection{Determination of protein concentration}

Protein concentration in antivenom preparations was determined by the Biuret reaction [17].

\subsection{Electrophoretic analysis}

Electrophoregram of the antivenom was obtained by polyacrylamide gel electrophoresis (SDS-PAGE) at a concentration of 7.5\% acrylamide under non-reducing conditions [18]. Gels were stained with Coomassie Brilliant Blue R-250.

\subsection{FPLC analysis}

Antivenom samples were analyzed by gel filtration fast-protein liquid chromatography (FPLC) in a Superdex 200 10/300 GL column (Amersham Biosciences, Sweden), using $20 \mathrm{mM}$ Tris-HCl, $150 \mathrm{mM}$ $\mathrm{NaCl}, \mathrm{pH} 7.5$ as eluent. The flow rate was $0.5 \mathrm{~mL} / \mathrm{min}$ [19].

\subsection{Hypersensitivity skin test}

Hypersensitivity skin test was performed by injecting intradermally $200 \mu \mathrm{L}$ of antivenom diluted 1:10 with isotonic saline solution, in the left side of the anal fold. As control, $200 \mu \mathrm{L}$ of isotonic saline solution were injected in the right side. Animals were observed for the next $30 \mathrm{~min}$. The diameters of swelling and erythema of test and control injection sites were recorded [9]. A skin test was interpreted as positive when the diameter of the swelling and erythema induced by the antivenom exceeds that induced by saline solution by $3 \mathrm{~mm}$.

\subsection{Administration of antivenom and clinical observation}

Forty seven healthy horses (300-350 kg body weight) and twenty healthy cows (200-250 kg body weight) were injected intravenously with equine antivenom at a dose of $0.4 \mathrm{~mL} / \mathrm{kg}$, which corresponds to a dose routinely used in the treatment of snakebite envenomations in these species (insert of polyvalent antivenom for veterinary use, Instituto Clodomiro Picado). Antivenom was administered undiluted into the jugular vein within $1 \mathrm{~min}$. Animals were meticulously observed during the following $3 \mathrm{~h}$ by a veterinarian for the appearance of noticeable manifestations of early adverse reactions, i.e. fever, diarrhea, urticaria, generalized rash, tachypnea or tachycardia [15]. In addition to the assessment of early adverse reactions, animals were observed on a daily basis during 40 days for the appearance of cutaneous manifestations (rash) characteristic of type III hypersensitivity reactions [20]. All procedures used in this study were approved by the Institutional Committee for the Care and Use of Laboratory Animals (CICUA) of Universidad de Costa Rica (project 82-08) and met the International Guiding Principles for Biomedical Research Involving Animals [21].

\subsection{Determination of anti-equine antibodies in the plasma of treated cows}

Blood samples were collected from the jugular vein immediately before and 40 days after the administration of antivenom in cows. Blood was allowed to clot at $20-22^{\circ} \mathrm{C}$ and serum was separated by centrifugation. To assess for the presence of bovine antibodies against equine $\operatorname{IgG}$, microplates were coated with $100 \mu \mathrm{L}$ of a $5 \mathrm{mg} /$ $\mathrm{dL}$ dilution of the antivenom. After washing the plates five times with $0.12 \mathrm{M} \mathrm{NaCl}, 0.04 \mathrm{M}$ phosphate buffer, pH 7.2 (PBS), $100 \mu \mathrm{L}$ of bovine serum samples, diluted 1:1000 in PBS-2\% bovine milk (BM), were added and the plates were incubated for $1 \mathrm{~h}$ at $20-22{ }^{\circ} \mathrm{C}$. Then, microplates were washed five times with PBS. Afterwards, $100 \mu \mathrm{L}$ of rabbit anti-bovine IgG conjugated with peroxidase, diluted 1:8000 with PBS-2\% BM, were added to each well. Again, microplates were incubated for $1 \mathrm{~h}$ at $20-22{ }^{\circ} \mathrm{C}$. After a final washing step, color development was performed by adding $150 \mu \mathrm{L}$ $\mathrm{H}_{2} \mathrm{O}_{2}$ and $o$-phenylenediamine (OPD), and the color reaction was stopped by the addition of $50 \mu \mathrm{L} 1.0 \mathrm{M} \mathrm{HCl}$. Absorbances were recorded at $492 \mathrm{~nm}$ and results were expressed as mean \pm SD $(\mathrm{n}=3)$. Determination of bovine antibodies against equine $\operatorname{Ig} \mathrm{G}$ was performed in 10 of the 20 cows studied.

\subsection{Determination of in vitro sensitization of bovine erythrocytes by equine natural antibodies present in the antivenom}

Before injection of antivenom, venous blood samples were collected from 10 of the 20 cows studied. A solution of $0.04 \mathrm{M}$ sodium citrate, $0.02 \mathrm{M}$ citric acid, $0.08 \mathrm{M}$ dextrose, pH 7.2 (ACD) was used as anticoagulant. After centrifugation, the erythrocyte pellet was washed five times with PBS. Then, a 5\% (v/v) erythrocyte suspension in PBS was prepared. Afterwards, $100 \mu \mathrm{L}$ of a $1: 10$ dilution of antivenom, or $100 \mu \mathrm{L}$ of PBS, were mixed with $100 \mu \mathrm{L}$ of erythrocyte suspension from each cow. The mixtures were incubated at $20-22{ }^{\circ} \mathrm{C}$ for $1 \mathrm{~min}$ and, after centrifugation at $500 \times \mathrm{g}$ for $30 \mathrm{~s}$, macroscopic agglutination was observed and graded, according with the intensity of the reaction, from "no agglutination" (-) to "very strong agglutination" (4+). Antiglobulin test was carried out by resuspending and washing the mixtures graded as "no agglutination" and mixing $100 \mu \mathrm{L}$ of each one with $100 \mu \mathrm{L}$ of rabbit anti-equine $\operatorname{IgG}$. After incubation at $20-22^{\circ} \mathrm{C}$ for $1 \mathrm{~min}$, the mixtures were centrifuged and observation of macroscopic agglutination was recorded as described. This experiment was controlled 
by repeating the same analysis, but substituting the rabbit antiequine $\operatorname{Ig} G$ with normal rabbit serum [22].

\subsection{Determination of in vivo sensitization of bovine erythrocytes by equine natural antibodies present in the antivenom}

Venous blood samples were collected from the cows before and $1 \mathrm{~h}$ after injection of antivenom, using ACD as anticoagulant. Erythrocytes from each sample were washed five times with PBS and suspensions at 5\% (v:v) in PBS were prepared. Then, $100 \mu \mathrm{L}$ of each suspension were incubated with $100 \mu \mathrm{L}$ of rabbit anti-equine IgG. After incubation at $20-22{ }^{\circ} \mathrm{C}$ for $1 \mathrm{~min}$, the mixtures were centrifuged and observation of macroscopic agglutination was recorded. This experiment was controlled by performing the same analysis, but substituting the rabbit anti-equine IgG with normal rabbit serum [22].

\subsection{Statistical analysis}

The significance of the differences between the mean values of the titres in samples from cows before and 40 days after antivenom administration was determined by the paired sample Student's $t$ test. A value of $p<0.05$ was considered to be significant.

\section{Results and discussion}

\subsection{Antivenom}

Early adverse reactions to antivenoms may be of two basic types, i.e. true anaphylactic reaction, mediated by IgE, or anaphylactoid reactions, mediated by mechanisms independent of IgE [1]. Traditionally, it has been accepted that the appearance of anaphylactoid reactions during the administration of antivenoms is associated with the anticomplementary activity of the formulations [6]. On this basis, it has been assumed that $\mathrm{F}\left(\mathrm{ab} \mathrm{b}_{2}\right)_{2}$-based formulations, which have lower anticomplementary activity than whole IgGbased formulations [23], would induce a lower incidence of anaphylactoid reactions. However, clinical evidence does not support this presumption since $\mathrm{F}\left(\mathrm{ab}^{\prime}\right)_{2}$ antivenoms induce early

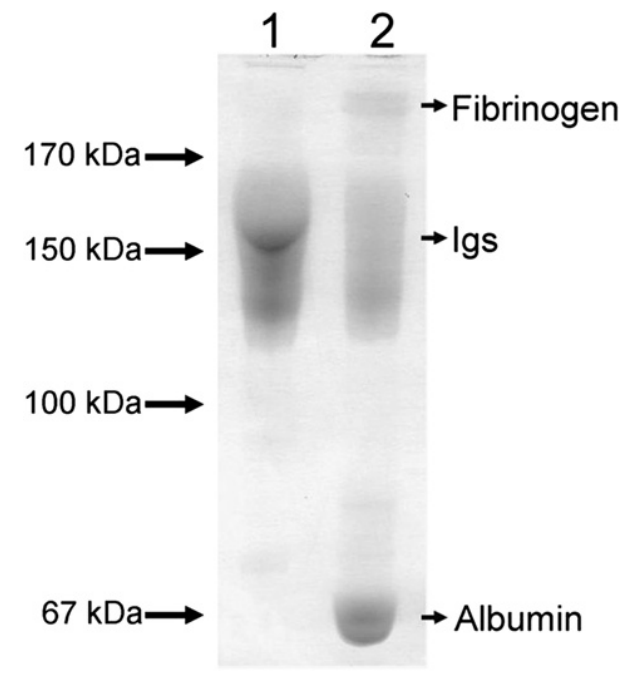

Fig. 1. Electrophoregram of equine-derived antivenom, constituted by whole IgG molecules. Non-reduced samples were loaded in a $7.5 \%$ polyacrylamide gel in the presence of SDS. Proteins were stained with Coomassie Brilliant Blue R-250. Lane 1 corresponds to purified antivenom, while lane 2 corresponds to equine plasma. Bands corresponding to fibrinogen, albumin and immunoglobulins are identified. adverse reactions with an incidence that varies depending on the particular product used $[11,12,15,24-28]$. This suggests that factors other than the in vitro anticomplementary activity of antivenoms must be involved in the genesis of anaphylactoid reactions.

Electrophoregram and FPLC analysis of the antivenom used in this study shows that: 1) more than $90 \%$ of the protein content corresponds to whole IgG, 2 ) less than $10 \%$ of the immunoglobulins occur as dimers or high molecular mass aggregates, and 3) this preparation has very low albumin content (Figs. 1 and 2). These results indicate that the antivenom used in this study has the physicochemical properties previously described for this formulation [13].

\subsection{Hypersensitivity skin test}

Hypersensitivity skin test is used to predict possible IgE-mediated anaphylactic reactions induced by a variety of medicines. However, since anaphylactoid reactions to antivenoms are not mediated by IgE, snake antivenom skin test has been of low value in predicting these reactions [9,29], and it is therefore not routinely recommended in the clinical administration of antivenoms [30,31]. Thus, intradermal skin test in antivenom administration is only valid for the prediction of type I hypersensitivity, i.e. anaphylactic reactions. In our study, hypersensitivity skin test was negative for all of the animals tested. This result means that anaphylactic reactions (type I hypersensitivity) were not expected to occur in the injected animals, a prediction that was fully corroborated by the experimental outcome (see below). These observations were expected since the cows used in this study had not been previously exposed to equine immunoglobulin and it is unlikely that horses would have IgE against their own IgG immunoglobulins without showing clinical manifestations.

\subsection{Antivenom administration and clinical observation}

In previous clinical trials performed in humans affected by snakebite envenoming, it was determined that intravenous administration of this antivenom formulation, at the same dose used in this study $(0.4 \mathrm{~mL} / \mathrm{kg})$, induces an incidence of anaphylactoid reactions of $15-20 \%[11,15]$. In contrast, our observations indicate that, during the following $3 \mathrm{~h}$ after the administration of

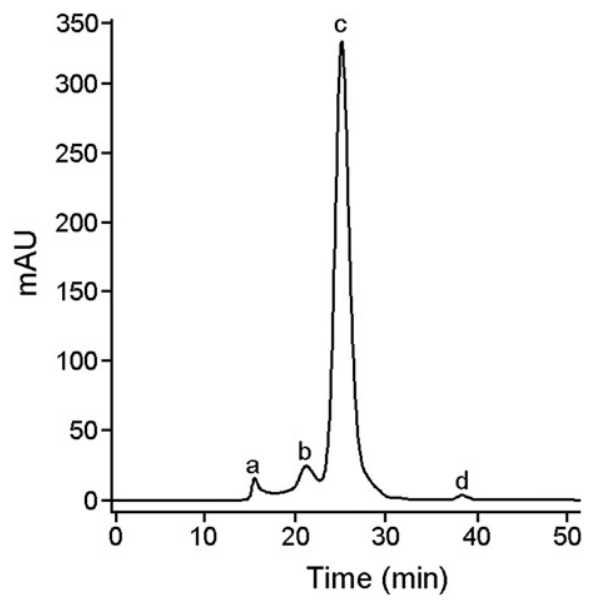

Fig. 2. Gel filtration fast-protein liquid chromatography (FPLC) profile of equinederived antivenom, constituted by whole IgG molecules. Antivenom samples were analyzed in a Superdex 200 10/300 GL column using $20 \mathrm{mM}$ Tris- $\mathrm{HCl}, 150 \mathrm{mM} \mathrm{NaCl}$ $\mathrm{pH} 7.5$ as eluent. The flow rate was $0.5 \mathrm{~mL} / \mathrm{min}$. Peaks labeled as a,b,c and d correspond to protein aggregates (2.5\%), immunoglobulin dimers (6.5\%), immunoglobulin monomers $(90.5 \%)$ and albumin $(0.5 \%)$, respectively. 


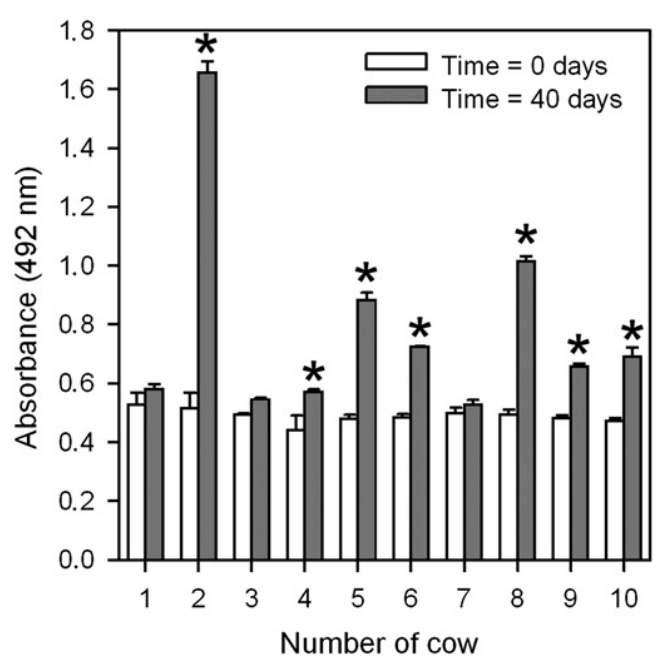

Fig. 3. Determination of bovine antibodies against horse immunoglobulins. ELISA plates were coated with $100 \mu \mathrm{L}$ of antivenom ( $5 \mathrm{mg}$ protein/dL). Then, $100 \mu \mathrm{L}$ of bovine serum samples, diluted 1:1000 in PBS-2\% bovine milk (BM), were added. Anti-equine antibodies were detected with a rabbit anti-bovine IgG conjugated with peroxidase, diluted 1:8000. Results are presented as the absorbances at $492 \mathrm{~nm}$ (mean \pm SD) of a 1:1000 serum dilution. Absorbance of the control of PBS- $2 \% \mathrm{BM}$ was $0.450 \pm 0.021$. *A significant increment $(P<0.001)$ in absorbance was observed between samples taken immediately before and 40 days after antivenom administration.

antivenom, none of the animals used in this study showed manifestations of early adverse reactions of any type. Moreover, no late adverse reactions, i.e. rash, were observed during a 40-day period after antivenom administration.

The difference between studies performed in humans and this study in animals could be attributed to the fact that a number of reactions such as nausea, headache and dizziness are readily recorded in humans, but not in animals. However, the majority of the clinical manifestations of anaphylactoid reactions, such as fever, vomiting, diarrhea, urticaria, generalized rash, tachypnea or tachycardia, which are easily detected in both human and animals, were not observed in our study, thus supporting the conclusion that early adverse reactions were absent in horses and cows after administration of this antivenom.

Another factor that must be considered is the fact that, unlike studies performed in envenomed humans, our present results were obtained in a group of non-envenomed individuals. Incidence of early adverse reactions induced by this antivenom formulation in envenomed animals has not been determined yet. The impact of this methodological difference on the incidence of early adverse reactions to antivenoms is unclear at present. Our results might be revealing a different tolerability towards equine-derived immunoglobulins in horses and cows as compared to humans.

Assuming that the incidence of anaphylactoid reactions to antivenom administration varies between different species, this agrees with the hypothesis that natural anti-species antibody responses of patients against foreign proteins are likely to be involved in the genesis of anaphylactoid reactions [7,8,32]. Thus, sensitization of animals and humans with heterologous horse immunoglobulins, through a variety of mechanisms different from therapeutic antibody administration, may explain the presence of heterophilic anti-horse IgG which may react with horse $\operatorname{IgG}$ upon antivenom infusion, provoking anaphylactoid reactions. From this point of view, it is not surprising that horses did not suffer anaphylactoid reactions when exposed to homologous antivenoms.

\subsection{Determination of anti-equine antibodies in the plasma of treated cows}

The genesis of anaphylactoid reactions could be related to heterophilic antibodies that occur naturally in the plasma of animals or humans towards the immunoglobulins that constitute the antivenom $[7,8,32]$. We found that cows have a basal antibody titre against equine $\operatorname{IgG}$, which is very similar in all animals tested (Fig. 3). Since no early adverse reactions were registered, it is clear that this basal anti-equine antibody titre is unlikely to play a relevant role in the pathogenesis of anaphylactoid reactions in cows. However, as shown in Fig. 3, 40 days after the administration of antivenom, the concentration of antibodies against equine IgG increased significantly $(t=-2.790, d f=9, P=0.021)$. Thus, equine antivenom IgG stimulates an antibody response in cows, a finding that may bear relationship to the potential development of a type III hypersensitivity reaction, i.e. serum sickness [20]. However, clinical inspection of animals did not reveal evidence of immune complex reactions, i.e. no rash development occurred, at the time antiequine antibody response developed.

\subsection{Determination of sensitization of bovine erythrocytes by antivenom antibodies}

Agglutination and lysis of erythrocytes by heterologous serum was described for the first time in 1875 by Leonard Landois [33].

Table 1

Agglutination of erythrocytes from non-envenomed cows, by equine-derived antivenom.

\begin{tabular}{|c|c|c|c|c|c|c|c|}
\hline \multicolumn{4}{|c|}{ in vitro sensitization } & \multicolumn{4}{|c|}{ in vivo sensitization } \\
\hline \multirow[t]{2}{*}{ Cow number } & \multirow[t]{2}{*}{ Direct agglutination $^{\mathrm{a}}$} & \multicolumn{2}{|l|}{ Before antivenom } & \multicolumn{2}{|l|}{ Before antivenom } & \multicolumn{2}{|l|}{ After antivenom } \\
\hline & & Antiglobulin test ${ }^{\mathrm{b}}$ & $\mathrm{NRS}^{\mathrm{c}}$ & Antiglobulin test & NRS & Antiglobulin test & NRS \\
\hline 1 & - & + & - & - & - & - & - \\
\hline 2 & - & + & - & - & - & - & - \\
\hline 3 & - & + & - & - & - & - & - \\
\hline 4 & - & + & - & - & - & - & - \\
\hline 5 & - & + & - & - & - & - & - \\
\hline 6 & - & + & - & - & - & - & - \\
\hline 7 & - & + & - & - & - & - & - \\
\hline 8 & - & + & - & - & - & - & - \\
\hline 9 & - & + & - & - & - & - & - \\
\hline 10 & - & + & - & - & - & - & - \\
\hline
\end{tabular}


Afterwards, it was demonstrated that serum haemolytic activity depends on the species used as complement, antibodies or erythrocytes sources [34]. Inability of complement system to lyse sensitized erythrocytes may be due either to: 1 ) non-activation of the complement system by the heterologous immunoglobulins, or 2 ) activation of the complement system over erythrocytes resistant to lysis [34].

Natural heterophilic anti-erythrocyte antibodies can be dragged along antivenom production from the initial plasma to the final product [22]. Therefore, administration of heterologous antivenoms to animals could result in intravascular haemolysis due to the action of such anti-erythrocyte antibodies. Also, a non-haemolytic complement fixation could result in anaphylatoxins release and consequently in the induction of the early adverse reactions observed during the antivenom administration.

As expected, the equine antivenom used in this study does not have antibodies against horse erythrocytes (not shown). However, it has natural antibodies against bovine erythrocytes. These antibodies are capable of mediating the agglutination of erythrocytes sensitized in vitro; nevertheless, due to their very low concentration, sensitization in vivo was undetectable in our experimental conditions (Table 1). These observations, together with the fact that no evident early adverse reactions occurred in cows, suggest that natural anti-bovine erythrocyte antibodies present in equinederived antivenom do not provoke adverse reactions in these bovines. Nevertheless, these findings cannot be extrapolated to other species and other animal sources of antivenom [34].

\section{Conclusions}

Polyvalent antivenom consisting of whole IgG purified from equine plasma by caprylic acid precipitation is routinely used in Central America in the treatment of viperid snakebite envenoming in humans and animals [35]. Our observations indicate that this antivenom is very well tolerated by horses and cows, i.e. no early adverse reactions occurred after bolus intravenous administration. Although it is necessary to extend these observations to envenomed horses and cows, our results and previous observations suggest that, in addition to the physicochemical characteristics of the formulation, other factors must determine the manifestation of adverse reactions in snakebite envenomed humans treated with equine-derived antivenoms. The conclusions reached in this study cannot be extrapolated to antivenoms manufactured using other animal sources of antivenom, and further investigations are necessary to assess this hypothesis.

\section{Conflict of interest}

There are no conflicts of interest.

\section{Acknowledgements}

This study was supported by Vicerrectoría de Investigación, Universidad de Costa Rica (project 741-A9-003) and CYTED (project 206AC0281). The authors thank our colleagues at Instituto Clodomiro Picado for their collaboration.

\section{References}

[1] Lalloo DG, Theakston RD. Snake antivenoms. J Toxicol Clin Toxicol 2003;41:277-90.

[2] Gutiérrez JM, León G. Snake antivenoms: technological, clinical and public health issues. In: UFMG, editor. Animal toxins: state of the art. Belo Horizonte: Sermograf; 2009. p. 393-421.

[3] Theakston RD, Warrell DA, Griffiths E. Report of a WHO workshop on the standardization and control of antivenoms. Toxicon 2003;41:541-57.
[4] Williams DJ, Jensen SD, Nimorakiotakis B, Müller R, Winkel KD. Antivenom use, premedication and early adverse reactions in the management of snake bites in rural Papua New Guinea. Toxicon 2007:49:780-92.

[5] Kobrynski LJ. Anaphylaxis. Clin Ped Emerg Med 2007;8:110-6.

[6] Sutherland SK. Serum reactions. An analysis of commercial antivenoms and the possible role of anticomplementary activity in de-novo reactions to antivenoms and antitoxins. Med J Aust 1977;1:613-5.

[7] Herrera M, León G, Segura A, Meneses F, Lomonte B, Chippaux JP, et al. Factors associated with adverse reactions induced by whole IgG preparations: comparison between horse, sheep and camel IgGs. Toxicon 2005;46:775-81.

[8] Sevcik C, Díaz P, D'Suze G. On the presence of antibodies against bovine equine and poultry immunoglobulins in human IgG preparations, and its implications on antivenom production. Toxicon 2008;51:10-6.

[9] Malasit P, Warrell DA, Chanthavanich P, Viravan C, Mongkolsapaya J, Singhthong B, et al. Prediction, prevention and mechanism of early (anaphylactic) antivenom reactions in victims of snake bites. Br Med J 1986;292:17-20

[10] Otero R, Cardoso JLC, Dias W, Higashi HG Nuñez V, Díaz A et al. A randomized blinded comparative trial of one pepsin-digested and two whole IgG antivenoms in Bothrops snake bites in Urabá, Colombia. Am J Trop Med Hyg 1998;58:183-9.

[11] Otero R, León G, Gutiérrez JM, Rojas G, Toro MF, Barona J, et al. Efficacy and safety of two whole IgG polyvalent antivenoms, refined by caprylic acid fractionation with or without $\beta$-propiolactone, in the treatment of Bothrops asper bites in Colombia. Trans R Soc Trop Med Hyg 2006;100:1173-82.

[12] Otero-Patiño R, Silva-Haad JJ, Barona MJ, Toro MF, Quintana JC, Diaz A, et al. Accidente bothrópico en Colombia: estudio multicéntrico de la eficacia y seguridad de Antivipmyn-Tri ${ }^{\circledR}$, un antiveneno polivalente producido en México. latreia 2007;20:244-62.

[13] León G, Lomonte B, Gutiérrez JM. Anticomplementary activity of equine whole IgC antivenoms: comparison of three fractionation protocols. Toxicon 2005;45:123-8.

[14] Rojas G, Jiménez JM, Gutiérrez JM. Caprylic acid fractionation of hyperimmune horse plasma: description of a simple procedure for antivenom production. Toxicon 1994;32:351-63.

[15] Otero R, Gutiérrez JM, Rojas G, Nuñez V, Díaz A, Miranda E, et al. A randomized blind clinical trial of two antivenoms, prepared by caprylic acid or ammonium sulphate fractionation of IgG, in Bothrops snake bites in Colombia. Correlation between safety and biochemical characteristics of antivenoms. Toxicon 1999;37:895-908

[16] Angulo Y, Estrada R, Gutiérrez JM. Clinical and laboratory alterations in horses during immunization with snake venoms for the production of polyvalent (crotalinae) antivenom. Toxicon 1997;35:81-90.

[17] Schosinsky K, Vargas M, Vinocour G, González OM, Brilla E, Gutiérrez A Manual de Técnicas de Laboratorio. Química Clínica. San José: Universidad de Costa Rica; 1983.

[18] Laemmli UK. Cleavage of structural proteins during the assembly of the head of bacteriophage T4. Nature 1970;227:680-5.

[19] García M, Monge M, León G, Lizano S, Segura E, Solano G, et al. Effect of preservatives on IgG aggregation, complement-activating effect and hypotensive activity of horse polyvalent antivenom used in snakebite envenomation. Biologicals 2002;30:143-51.

[20] LoVecchio F, Klemens J, Roundy EB, Klemens A. Serums sickness following administration of Antivenin (Crotalidae) Polyvalent in 181 cases of presumed rattlesnake envenomation. Wilderness Environ Med 2003;14:220-1.

[21] Council of International Organizations of Medical Sciences (CIOMS). The international guiding principles for biomedical research involving animals. Geneva: Z.Bankowski \& N. Howard-Jones; 1986.

[22] León G, Rodríguez M, Rucavado A, Fernandez I, Lomonte B, Gutiérrez JM. Antihuman erythrocyte antibodies in horse-derived antivenoms used in the treatment of snakebite envenomations. Biologicals 2007;35:5-11.

[23] León G, Monge M, Rojas E, Lomonte B, Gutiérrez JM. Comparison between IgC and $\mathrm{F}\left(\mathrm{ab}^{\prime}\right)_{2}$ polyvalent antivenoms: neutralization of systemic effects induced by Bothrops asper venom in mice, extravasation to muscle tissue, and potential for induction of adverse reactions. Toxicon 2001;39:793-801.

[24] Moran NF, Newman WJ, Theakston RDG, Warrell DA, Wilkinson D. High incidence of early anaphylactoid reaction to SAIMR polyvalent snake antivenom. Trans R Soc Trop Med Hyg 1998;92:69-70.

[25] Hui FW, Marcopito LF, Cardoso JLC, França FOS, Malaque CMS, Ferrari RA, et al Sequential randomized and double blind trial of promethazine prophylaxis against early anaphylactic reactions to antivenom for Bothrops snake bites. $\mathrm{Br}$ Med J 1999;318:1451-3.

[26] Premawardhena AP, de Silva CE, Fonseka MMD, Gunatilake SB, da Silva HJ. Low dose subcutaneous adrenalin to prevent acute adverse reactions to antivenom serum in people bitten by snakes: randomised, placebo controlled trial. Br Med J 1999;318:1041-3.

[27] Ariaratnam CA, Sjostrom L, Raziek Z, Kularatne AM, Arachichi RWK, Sheriff MHR, et al. An open, randomized comparative trial of two antivenoms for the treatment of envenoming by Sri Lankan Russell's viper (Daboia russelii russelii). Trans R Soc Trop Med Hyg 2001;95:74-80.

[28] Gawarammana IB, Kularatne SAM, Dissanayake WP, Kumarasiri RPV, Senanayake N, Ariyasena H. Parallel infusion of hydrocortisone \pm chlorpheniramine bolus injection to prevent acute adverse reactions to antivenom for snakebites. A randomized, double-blind, placebo-controlled study. Med J Aust 2004:180:20-3.

[29] Rojnuckarin P. Antivenom skin test: theory versus practice. Acta Trop 2009;109:86. 
[30] Warrell DA. WHO/SEARO guidelines for the clinical management of snake bites in the southeast Asian region. Southeast Asian J Trop Med Public Health 1999;30:1-85

[31] Cupo P, Azevedo-Marques MM, Sarti W, Hering SE. Proposal of abolition of the skin sensitivity test before equine rabies immune globulin application. Rev Inst Med Trop São Paulo 2001;43:51-3.

[32] León G, Segura A, Herrera M, Otero R, França FOS, Barbaro KC, et al. Human heterophilic antibodies against equine immunoglobulins: assessment of their role in the early adverse reactions to antivenom administration. Trans $\mathrm{R}$ Soc Trop Med Hyg 2008;102:1115-9.
[33] Hughes NC, Gardner B. Red cell agglutination: the first description by Creite (1869), and further observations made by Landois (1875) and Landsteiner (1901). Br J Haematol 2002;119:889-93.

[34] Grant CK. Complement "specificity" and interchangeability: measurement of hemolytic complement levels and use of the complement-fixation test with sera from common domesticated animals. Am J Vet Res 1977;38:1611-7.

[35] Gutiérrez JM. Snakebite envenomation in Central America. In: MacKessy SP, editor. Handbook of venoms and toxins of reptiles. Boca Raton: CRC Press; 2009. p. 491-507. 